\title{
Tuning the Spatially Controlled Growth, Structural Self-Organizing and Cluster-Assembling of the Carbyne-Enriched Nanostructured Metamaterials During Ion-Assisted Pulse-Plasma Deposition
}

\author{
Alexander Lukin ${ }^{1}$, Oğuz Gülseren ${ }^{2}$ \\ ${ }^{1}$ Western-Caucasus Research Center, Tuapse, 352808, Russian Federation \\ ${ }^{2}$ Department of Physics, Bilkent University, Ankara, 06800, Turkey
}

Corresponding Author: lukin@wcrc.ru

\begin{abstract}
Structural self-organizing and pattern formation are universal and key phenomena observed during growth and cluster-assembling of the carbyne-enriched nanostructured metamaterials at the ionassisted pulse-plasma deposition. Fine tuning these universal phenomena opens access to designing the properties of the growing carbyne-enriched nano-matrix. The structure of bonds in the grown carbyneenriched nano-matrices can be programmed by the processes of self-organization and auto-synchronization of nanostructures. We propose the innovative concept, connected with application of the universal Cymatics phenomena during the predictive growth of the carbyne-enriched nanostructured metamaterials. We also propose the self-organization approach for increase stability of the long linear carbon chains. The main idea of suggested concept is manipulating by the self-organized wave patterns excitation phenomenon and their distribution by the spatial structure and properties of the nanostructured metamaterial grows region through the new synergistic effect. Mentioned effect will be provided through the vibration-assisted self-organized wave patterns excitation along with simultaneous manipulating by their properties through the electric field. We propose to use acoustic activation of the plasma zone of nano-matrix growing. Interaction between the inhomogeneous electric field distribution generated on the vibrating layer and the plasma ions will serve as the additional energizing factor controlling the local pattern formation and self-organizing of the nanostructures. Suggested concept makes it possible to provide precise predictive designing the spatial structure and properties of the advanced carbyne-enriched nanostructured metamaterials.
\end{abstract}

Keywords: carbyne-enriched nanostructured metamaterials; sp1-hybridized bonds; carbon atomic wires; ion-assisted pulsed-plasma deposition; nanocavities; cluster-assembling; vibration-assisted growing; acoustic activation; vibrational patterns; electromagnetic activation; self-organizing of the nanostructures; nano-cymatics; controlled patterning; synergistic effect

\section{Introduction}

Ideal one-dimensional form of carbon, a carbon allotrope - an infinitely long the linear chain of carbon atoms, named as a carbyne, attracted much interest due to advanced mechanical and physicochemical properties, including mechanical strength predicted to be an order of magnitude higher than that of diamond, [1 - 3]. 
Like a graphene, carbyne is just one atom thick, which gives it an extremely large surface area in relation to mass.

Back in the 1930s, astronomers discovered carbynes as one of the first molecules in the interstellar space. In this connection, carbyne can be considered as is out-of-this-world interstellar material. Later, astronomers have found the signs of the presence of cosmic carbyne crystals in interstellar dust clouds and in the material of some carbonaceous meteorites.

Unique combination of extraordinary properties of the carbyne-enriched nanostructured metamaterials offers a fascinating material platform for the development of next-generation technologies in many areas - superfast electronics, ultrasensitive sensors, multifunctional composites \& coatings, membranes, medicine \& biotechnology, energy storage matrices like batteries and supercapacitors, where the surface area of the electrode determines energy density. The list of key application areas of the carbyne-enriched nanostructured metamaterials is shown in Figure 1.

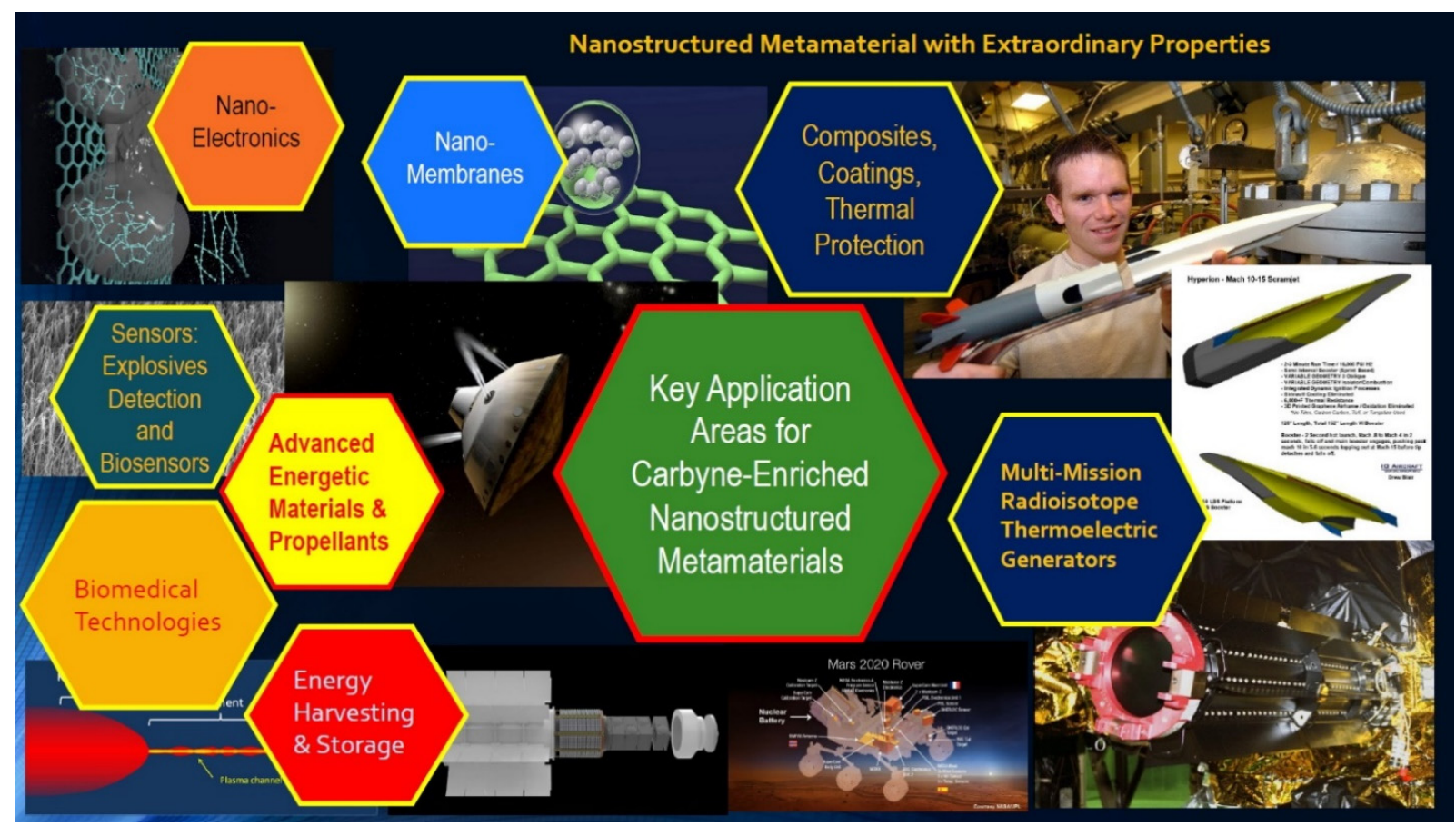

Figure 1. Key application areas for carbyne-enriched nanostructured metamaterials. 
Among such applications are the following: - electronic thermal protection systems; functionalized nano-sized additives for energetic materials and propellants; - metamaterials for supercapacitor applications; - next generation of solar cells based on carbyne-enriched metamaterials; - carbyne-enriched metamaterials for multi-mission radioisotope thermoelectric generators; - carbyne-enriched metamaterials for active nano-membranes that allow continuous tuning of their functional parameters to target molecules by changing the parameters of the electric field in the pores. Carbyne-enriched nano-structures are critical for sensing applications, as they have unique physical and electronic properties which permit detection of substances in solutions, gaseous compounds and pollutants.

The properties of the carbon-based nanostructured metamaterials essentially depend on their spatial structure. Differently hybridized carbon atoms are capable to form diamond, graphite, linear-chain grids and many other specific allotropes. Each carbon allotrope has notably different electronic and mechanical properties.

The electronic structure of a linear-chain carbon molecule includes two kinds of bonds: the sigma $(\sigma)$ bond provides mechanical stability to the linear-chain carbon molecule, and the ( $\pi$ ) bond, in addition to mechanical stability, provides the electrical properties of this nanostructure, since the $\pi$-electrons are delocalized and belong to the entire chain of atoms.

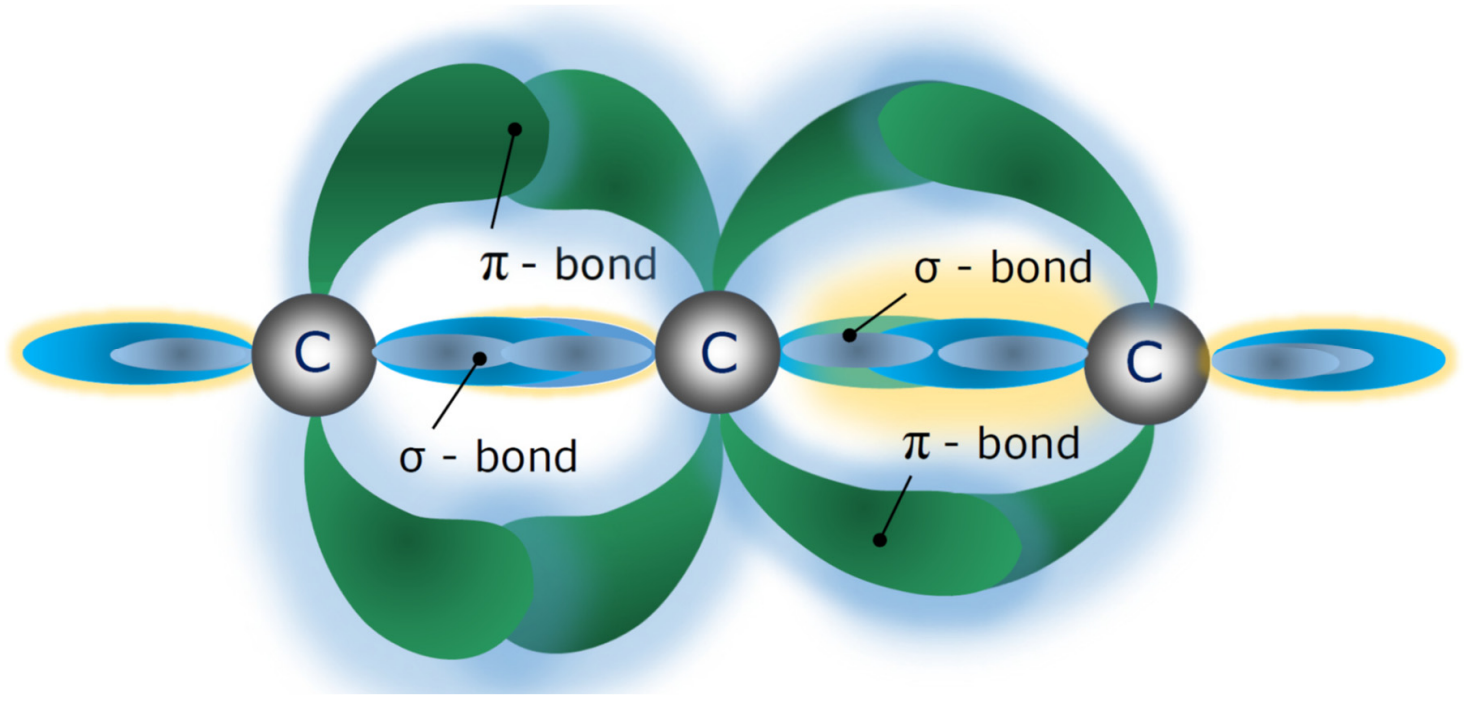

Figure 2. The electronic structure of a fragment of a linear-chain carbon molecule section, [3]. 
Carbon allotropes can function either as a conductor, in sp-1 chains, carbynes, and sp-2 planar structures, graphene and graphite, or as a wide-gap insulator in sp-3 tethrahedral coordination e.g., diamond and alkanes. The first two have the potential to form bonds that are electrically conducting, while sp-3 has insulating properties. For instance, carbon nanotube demonstrates remarkable electrical conductivity and anisotropic electromagnetic characteristics.

The existence of the cosmic carbyne can be used as a key to it formation mechanism and environments experienced by it and additionally confirms the presence of a factor of selforganization in the carbon chains growth. Linear chains are present in carbon vapors at temperatures above $5000 \mathrm{~K}$.

The technology for growing this unique carbon nanostructured metamaterial is enough simple: it self-organizes during condensation from the carbon vapor in a vacuum at temperature above $3150 \mathrm{~K}$.

However, pure carbyne in the condensed phase is an extremely unstable due to it high chemical reactivity. The growth of the macroscopic crystals of the carbyne is inhibited by the instability and high reactivity of this allotropic form of carbon. Carbyne properties can be manipulated through the chain length, doping by nano-clusters, and by type of the chain termination. Nanostructure's stability depends from the linear carbon chains length. Free CAWs of any length must be terminated by molecular complexes to ensure their stability.

For practical use of carbyne-enriched nanostructured metamaterials, the ability to ensure high stability of this nanomaterial is of key importance. Most of current research efforts concentrated on searching possibilities for stabilization of the sp-hybridized carbon chains.

\section{Stabilization of the sp-Hybridized Carbon Chains}

During a long time attempts to growth the long carbyne molecules were limited by its extreme chemical instability, [3]. In 2016, scientists of University of Vienna have proposed and demonstrated a fundamentally new strategy to ensure the structure stability of the extremely long sp-hybridized carbon chains, containing more than 6000 carbon atoms through growing them within the long nano-matrices formed by the double-walled carbon nanotubes, [4, 5], (Fig. 3). 


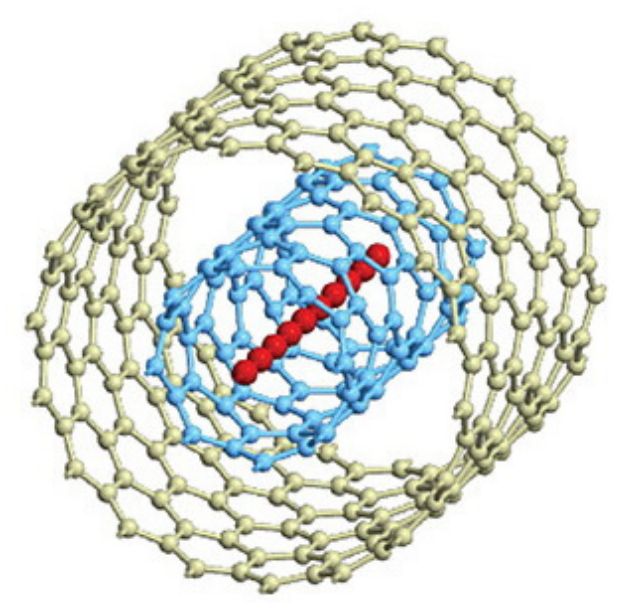

Figure 3. Schematic representation of one of examples of stabilizing of the long onedimensional carbyne molecule inserted into the double-walled carbon nanotube, $[4,5]$.

The double-walled carbon nanotubes provide efficient structure stabilizing of the encapsulated one-dimensional carbyne molecule that is extremely important for designing the advanced functional nanostructures metamaterials. Such kind of carbon nanotubes serve as nanoreactors and protect the sp-hybridized carbon chains from interaction with the environment. This outstanding result showed the new fundamental possibility of using the control of the nanomatrix spatial structure for programming the stability of the inserted sp-hybridized carbon chains.

The strategy, connected with the growth of the sp-hybridized carbon nanostructures in the composition of multi-cavity nano-matrices seems to be the most promising way for the creation of the advanced carbon-based nanostructured metamaterials.

The new alternative modification of this strategy, suggested in 2019, was connected with designing a new class of carbon allotropes obtained by combining linear sp-hybridized carbon chains with sp 3 bulk carbon through inserting of sp-hybridized chains into cylindrical nanocavities periodically arranged in hexagonal diamond, [6].

Schematic representation of one of examples of stabilizing of the of $\mathrm{sp}$-hybridized carbon chains through inserting into cylindrical nanocavities periodically arranged in hexagonal diamond is shown in Figure 4. 

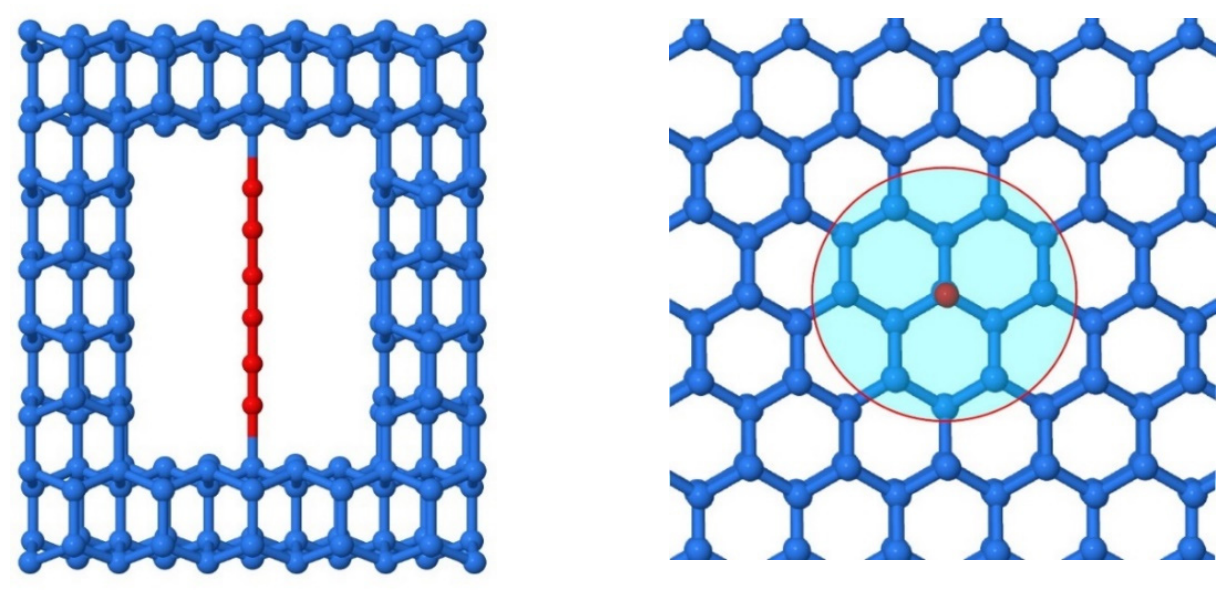

Figure 4. Schematic representation of one of examples of stabilizing of the of sp-hybridized carbon chains (red-highlighted sp atoms) through inserting into cylindrical nanocavities periodically arranged in hexagonal diamond (blue-highlighted $\mathrm{sp}^{3}$ carbon): a side view and a top cut, [6].

Since the sp-hybridized carbon chains are highly unsaturated, they have tendency to react with forming the extra bonds that leads to degrading into both $\mathrm{sp}^{2}$ and $\mathrm{sp}^{3}$ structures. In this connection, it is possible to provide inserting and isolation of the sp-hybridized carbon chains inside the some enough both wide and long nanocavity, formed by the $\mathrm{sp}^{3}$ hybridized carbon, to prevent the sp-hybridized carbon chains spontaneous reaction and degrading. As such an insulating nanostructure was chosen the hexagonal-structured diamond that have enough long nanocavity along the axis.

In the results was growed allotrope demonstrating characteristic high-frequency vibrations associated with the sp-hybridized chain stretching modes and having long-time stability at the room temperature environments.

One of promising routes for obtaining of the stable carbyne-enriched nanostructured metamaterials is growing them within the composition of multi-cavity nano-matrixes through the ion-assisted pulse-plasma deposition from the carbon plasma, [7].

This technique also opens possibilities for further increase of the long carbon chains stability through assembling them by atomic clusters of different chemical elements, for instance, Silver, Gold, Titanium, etc.

Due to outstanding deposition possibilities: the layer-by-layer deposition, possibility of 
metastable carbon phase synthesis, low substrate heating and applicability for various substrate types, this tool-kit continues studied Worldwide.

Such kind of carbon nanostructured metamaterials were named as two-dimensionally ordered linear-chain carbon, which is a two-dimensionally packed hexagonal array of carbon chains held by the van der Waals forces with the interchain spacing approximately being between (4.8-5.03) angstroms, [7]. Within such nano-matrix the carbon atom wires very weakly interacting with each other (due to Van der Waals interaction), and therefore, the properties of such nanomatrices are actually determined by the properties of individual quantum wires. The ordered array of the one-dimensional carbon chains packed parallel to one another in hexagonal structures are oriented perpendicular to the substrate surface. Geometric characteristics of the two-dimensionally ordered linear-chain carbon nano-matrix is presented on Figure 5 .
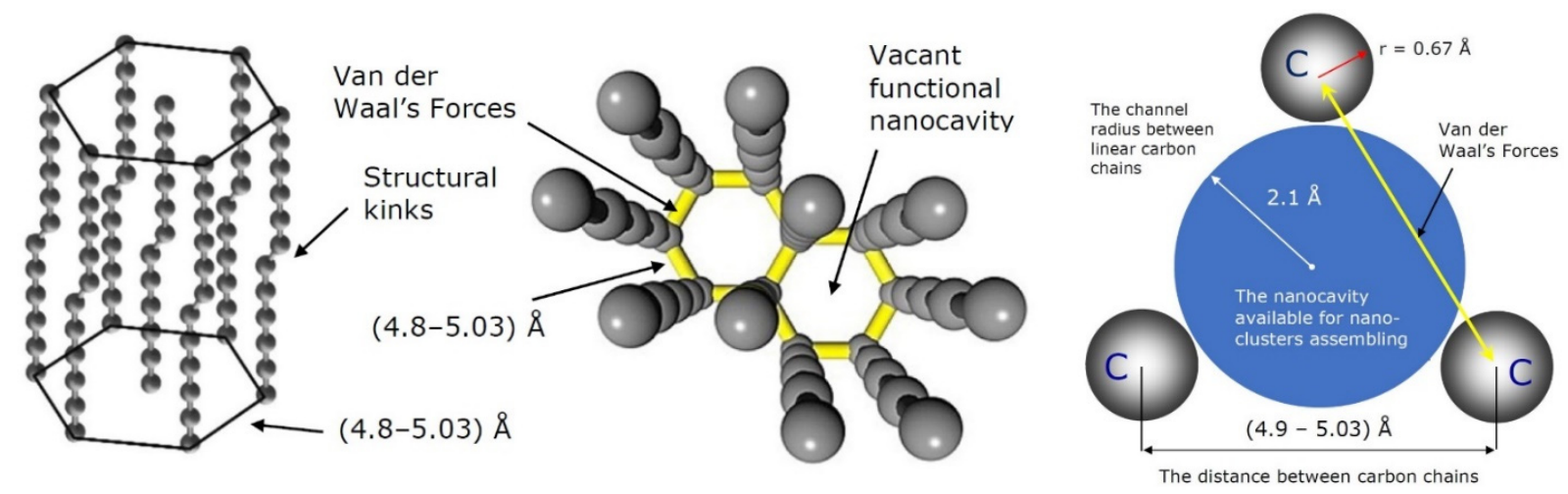

Figure 5. Geometric characteristics of the two-dimensionally ordered linear-chain carbon nanomatrix cell with the vacant functional nanocavity, available for the atomic nano-clusters assembling.

Along the vertical axis, the electrical conductivity of the nano-matrix is metallic. Due to absence of coupling between the carbon chains along the longitudinal and transverse axes (where the Van der Waals forces only exists) the nano-matrix demonstrate dielectric properties along these axes.

Such kind of nano-matrix can be considered as an array of mutually-stabilizing CAWs, which also includes a set of different sp-phases that provides stabilization of the sp-hybridized carbon chains. 
Two-dimensionally ordered linear-chain carbon nano-matrix represent a multi-cavity nanostructure, containing a vacant functional nanocavities, available for assembling by atom clusters of various chemical elements, (Fig. 5). By cluster-assembling of the spatial structure of two-dimensionally ordered linear-chain carbon nano-matrix with various molecules, specific catalytic agents and chemical elements, the properties of the nano-matrix can be both modified or the new properties can be added. Cluster-assembling of the nano-matrix can occur both without chemical interaction (so called intercalation), and with rupture of the $\pi$ bonds, which can lead to addition reaction. For instance, by assembling the two-dimensionally ordered linearchain carbon nano-matrix with calcium clusters, which suck up hydrogen molecules, creates a high-density, reversible hydrogen storage device.

The two-dimensionally ordered linear chain carbon nano-matrix could serve as an efficient basis for designing and the growth of the new carbon-based nanostructured metamaterials with unique electrophysical, optical, structural, topographic, biological and chemical properties. The spatial structure of such kind of nano-matrix can also self-adjusts to the structure of the embedded atom clusters.

\section{Pulse-Plasma Deposition Reactor for Growing of the Carbyne-Enriched Nanostructures}

The cathodic arc plasma deposition (CAPD) or arc-PVD (PVD is physical vapor deposition) is a physical vapor deposition technique in which an electric arc is used to vaporize material from a cathode target. The vaporized material then condenses on a substrate, forming a twodimensionally ordered linear-chain carbon nano-matrix.

The experimental set-up for ion-assisted pulse-plasma deposition of the two-dimensionally ordered linear-chain carbon nano-matrix with capability of cluster-assembling by various chemical elements are presented at the Figure 6 . The main components of the carbon pulseplasma deposition reactor for growing of the carbyne-based nanostructures: - vacuum chamber; - pulse-plasma carbon generator; - the ion source for ionic stimulation; - target assembly with removable target material.

The ion and plasma beams intersect at the substrate surface. The ion beam irradiation of the substrate surface forms bends in the attached carbon chains which stabilize the growing chain ensemble. 


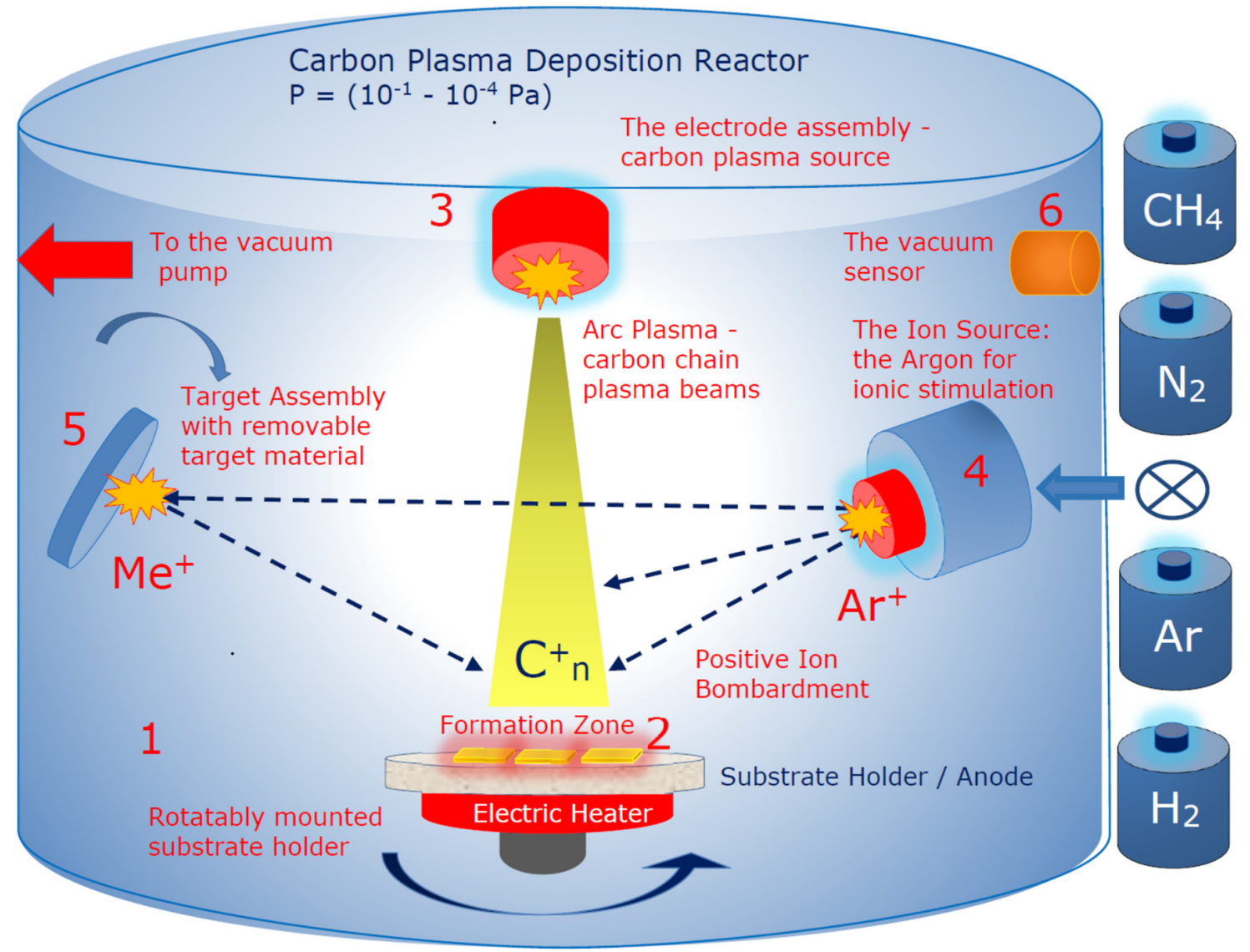

Figure 6. Schematic representation of the carbon pulse-plasma deposition reactor for growing of the carbyne-based nanostructures: 1 - vacuum chamber; 2 - substrate; 3 - pulse-plasma carbon generator (graphite cylindrical cathode of main discharge); $4^{-}$the ion source for ionic stimulation; 5 - target assembly with removable target material; 6 - vacuum sensor.

The evaporation of the carbon plasma sheaf from the graphite cathode of main discharge 3 is caused by local heating of the graphite surface by electron bombardment to $T=3000^{\circ} \mathrm{C}$. The chains of carbon atoms, $C_{n}$ (where $n=1,2,3, \ldots$ ), formed in the plasma sheaf are directed by electrodes to impinge upon the surface of the substrate where the polycondensation of the carbon chains takes place. The schematic representation of the pulse-plasma carbon generator that installed in the reactor of the experimental set-up (Figure 6) are presented at the Figure 7. 


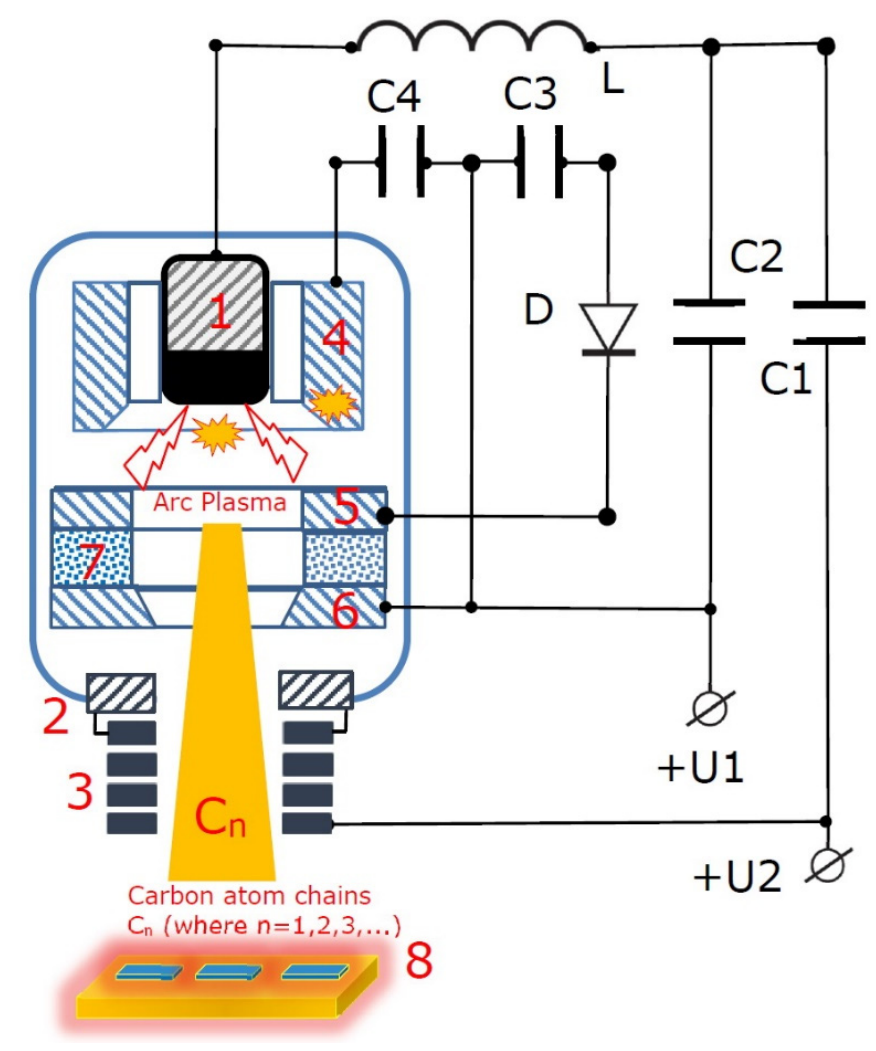

Figure 7. Schematic representation of the pulse-plasma carbon generator that installed in the reactor of the experimental set-up (Fig. 6): 1 - a cylindrical cathode of main discharge (evaporated material, the high purity graphite); 2 - anode of the main discharge; 3 - a solenoid final focusing system with plasma neutralization; 4 - second anode of auxiliary discharge; 5 ignition electrode; 6 - cathode of auxiliary discharge; 7 - dielectric insulator; 8 - substrate holder.

An arc discharge is ignited between the cathode of main discharge 1 and anode of main discharge 2 (which are preferably separated by a voltage of about $200 \mathrm{~V}$ ) by means of auxiliary discharge between the cathode of auxiliary discharge 6 and the cathode of main discharge 1 and the anode of auxiliary discharge 4 surrounding the cathode of main discharge 1 . The auxiliary discharge is ignited by means of ignition electrode 5. The design of the cathode (item 1 in Fig. 7) depends from the purposes of deposition and can be manufactured as a composite structure, containing cylindrical rods from different materials, for instance, silver, tungsten, gold etc. The example of design of a cylindrical cathode of main discharge is presented on Figure 8. 


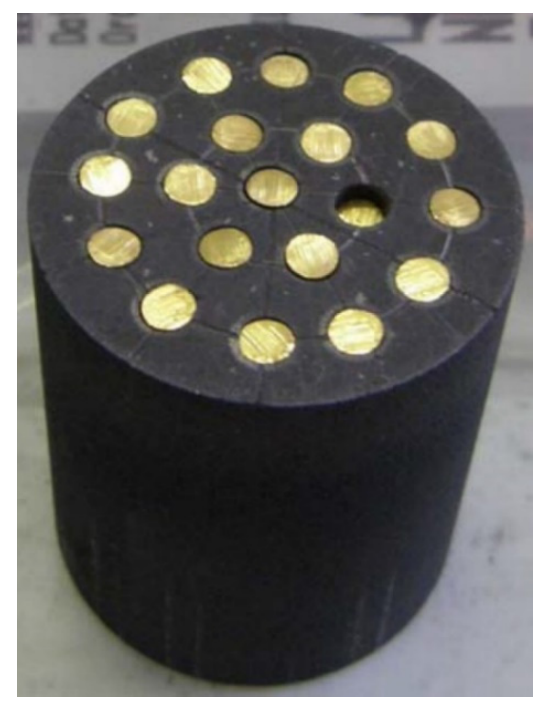

Figure 8. The example of design of a cylindrical cathode of main discharge, containing cylindrical rods from different materials, for instance, silver, tungsten, gold etc. This is item 1 n Figure 7, [8].

The capacitors $C_{1}$ and $C_{2}$ are connected to a power supply unit with varied voltage (100-300 V). The ignition electrodes are supplied by the pulsed voltage with an amplitude of $800 \mathrm{~V}$. The inductance $L$ are used for reduce the current growth rate to a required value. The growth of the carbon nano-matrix is enhanced by irradiation with Ar ions. The flux of Ar ions is formed by a lowpressure ion source, installed in a separate section of the deposition reactor chamber, (Fig. 6). The energy of ions bombarding the substrate surface depends on the substrate bias voltage, being varied in the range $0-300 \mathrm{eV}$ by both the carbon plasma parameters and the ion source extractor voltage, depending on the parameters of the plasma assisted deposition. The nanomatrix can be deposited onto Si wafer, metals, and $\mathrm{NaCl}$ single crystals at an ion energy of 150 $\mathrm{eV}$. Before deposition, the reactor chamber was pumped down to the residual pressure of $10^{-4}$ $\mathrm{Pa}$. The operating pressure during the deposition is $10^{-4} \mathrm{~Pa}$. The structure of bonds in the grown carbon nano-matrixes can be programmed by the processes of self-organization and autosynchronization of the growing nanostructures.

As a number of experiments show the $\mathrm{sp}^{1}$-bonds and $\mathrm{sp}$-hybridized carbon nanostructures are formed only in a narrow range of optimal ion-assisted pulse-plasma deposition parameters. Hence, a challenging task is to simulate and optimize a ion-assisted pulse-plasma deposition procedure. 


\section{Pattern Excitation Phenomena in the Model Experimental Systems}

Pattern's excitation phenomena in the pulse-plasma deposition zone are programmed by interaction of several competing mechanisms, in particular, through the thermo-electric convection excitation, by the state of stress in the deposited nano-matrix and by selfsynchronization of the self-excited oscillatory cells in the deposition region. Structural selforganization and pattern formation are the universal and key phenomena observed during growth and cluster-assembling of the carbyne-based nano-matrix at the ion-assisted pulseplasma deposition. Self-organization phenomena are observed also during the clusterassembling of the nano-cavities by atoms of various chemical elements due to formation of new chemical, interatomic and intermolecular bonds. The pulse-plasma deposition zone is a vibration-sensitive media for which the universal laws of Cymatics are valid, [9].

Let's consider the examples of the self-organizing and pattern excitation phenomena for the model experimental systems. The examples of self-organized patterns excitation for the samples growed at various ion assistance energy is presented in Fig. 9, [10].

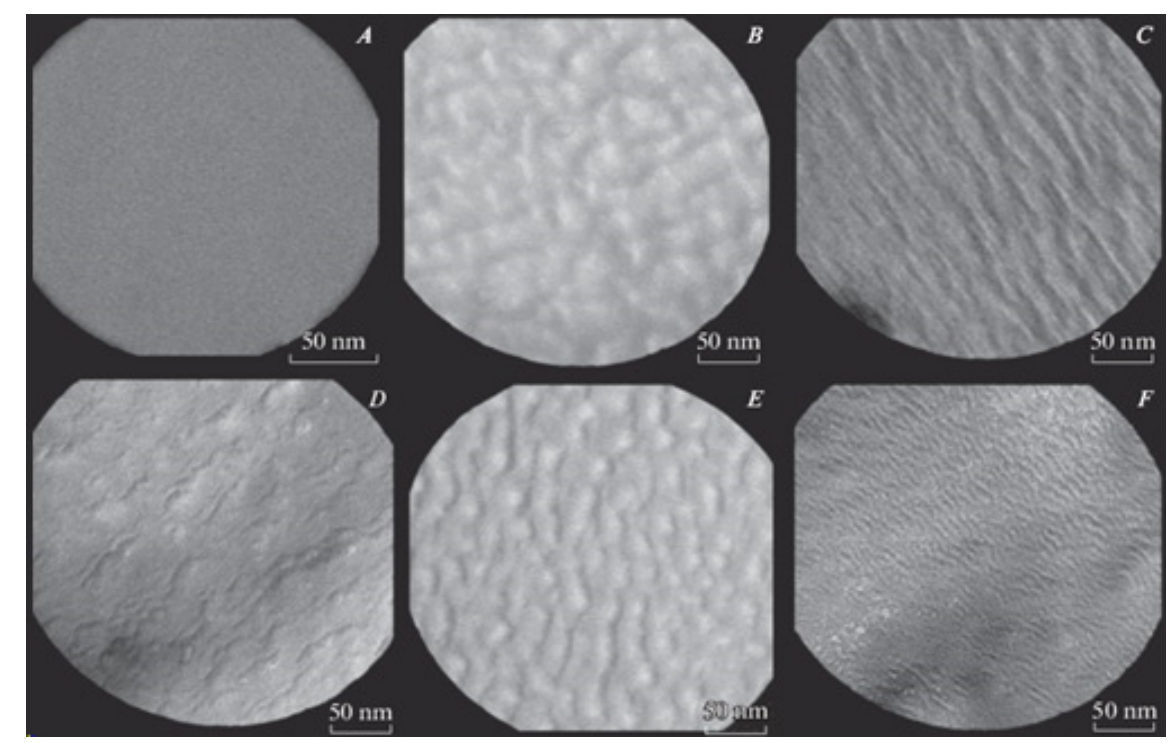

Figure 9. Transmission electron microscopy (TEM) patterns of samples obtained in different conditions: A - o eV, B - $200 \mathrm{eV}, \mathrm{C}-300 \mathrm{eV}, \mathrm{D}-400 \mathrm{eV}, \mathrm{E}-600 \mathrm{eV}, \mathrm{F}-800 \mathrm{eV},[10]$

Self-organization and formation of surface patterns are most pronounced when the growed system is supplied with additional energy and by nano-sized active centers. The transmission electron microscopy has shown that structure of the sample deposited without ion assistance is 
homogeneous, while the structure of ion-assisted samples is heterogeneous. The specific conductivity of ion-assisted samples is $10^{3}-10^{4}$ times larger than the conductivity of the samples deposited without ion assistance.

The transmission electron microscopy for case of assembling the two-dimensionally ordered linear chain carbon nano-matrix by silver nanoclusters has shown, that at increase of the energy flux into the deposition region occurs decrease of the average size of the active nucleation centers at simultaneous increase in their numbers, [11].

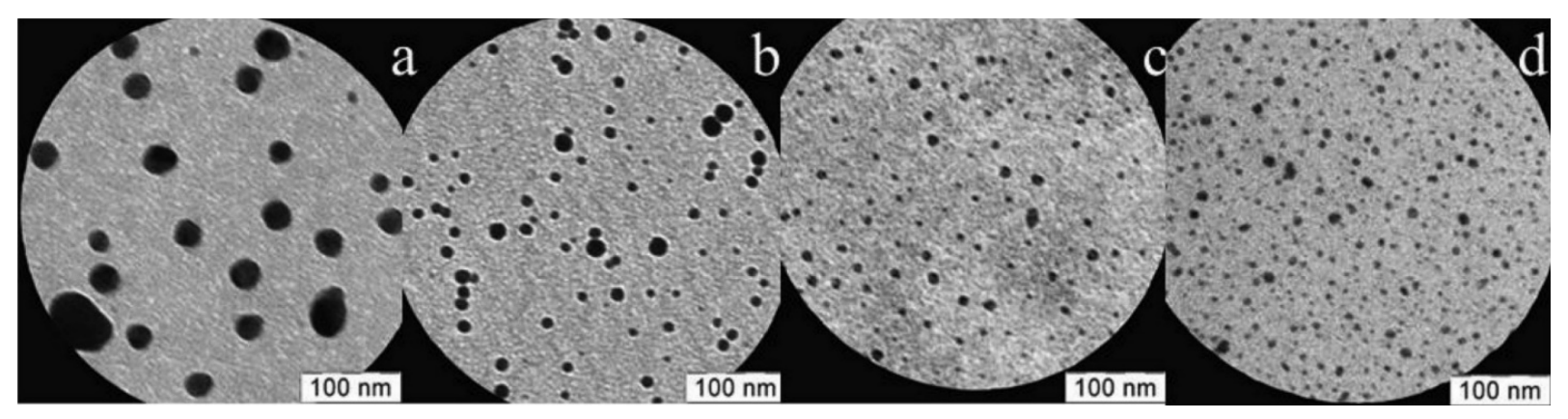

Figure 10. Transmission electron microscopy (TEM) images of the samples deposited at different ion assistance energies: (a) o eV, (b) $100 \mathrm{eV}$, (c) $200 \mathrm{eV}$, (d) $300 \mathrm{eV}$, [11].

\section{Model Experimental Systems for Study of Vibration-Assisted Deposition}

The model experimental system demonstrates how vibrations provide significant influence on the structures of the deposited model nanomaterial. In the paper [12] demonstrated a new approach of the evaporated material vibration-assisted thermal deposition in vacuum chamber. Earlier, in the papers $[13,14]$ for the atomic deposition experiment were studied the nanoscale self-organization phenomena on the substrate surface with using standing surface acoustic wave. With using molecular dynamics simulations were described the structuration physical driving mechanism.

However, this research does not take into account the vacuum conditions influence during the deposition processes. Acoustic waves cannot propagate in the low-pressure environment. At the same time, the acoustic waves can be transmitted from the vibration source to the solid as mechanical vibrations with specific frequency. In the result, the substrate mechanical vibrations during the deposition could influence on the surface morphology and structure of the deposited nanomaterial. 
The selenium, having a variety of allotropic phases, is a convenient material for fundamental researches of the mechanical vibrations influence during the nanostructure's deposition. Tellurium, as well as hexagonal selenium, is a typical crystalline semiconductor, whose atoms form polymeric, covalently bonded helical chains, packed into a hexagonal lattice through the van der Waals forces. Due to this specific nature of tellurium, during nanostructures deposition formation of the nanowires, nanotubes, nanorods, etc. is observed. Such kind of specific nanostructures can be considered as the model experimental systems that can demonstrates fundamental phenomenon observed at vibration-assisted thermal deposition of the evaporated material in vacuum with acoustic wave frequency. During the nano-structures deposition, the mechanical oscillations were applied to the substrate with input frequencies of $0,50,150$ and 4 $\mathrm{kHz}$ at the deposition rate of $0.3 \mathrm{~nm} / \mathrm{s}$, and the vacuum chamber pressure of $7 \times 10^{-3} \mathrm{~Pa}$.

As can be seen from the atomic force microscopy (AFM) images of $150 \mathrm{~nm}$ thick Tellurium nanostructures, presented on the Fig. 11, the acoustic waves applied to the substrate results in morphological changes, demonstrated self-organizing of the nanostructures, [12].
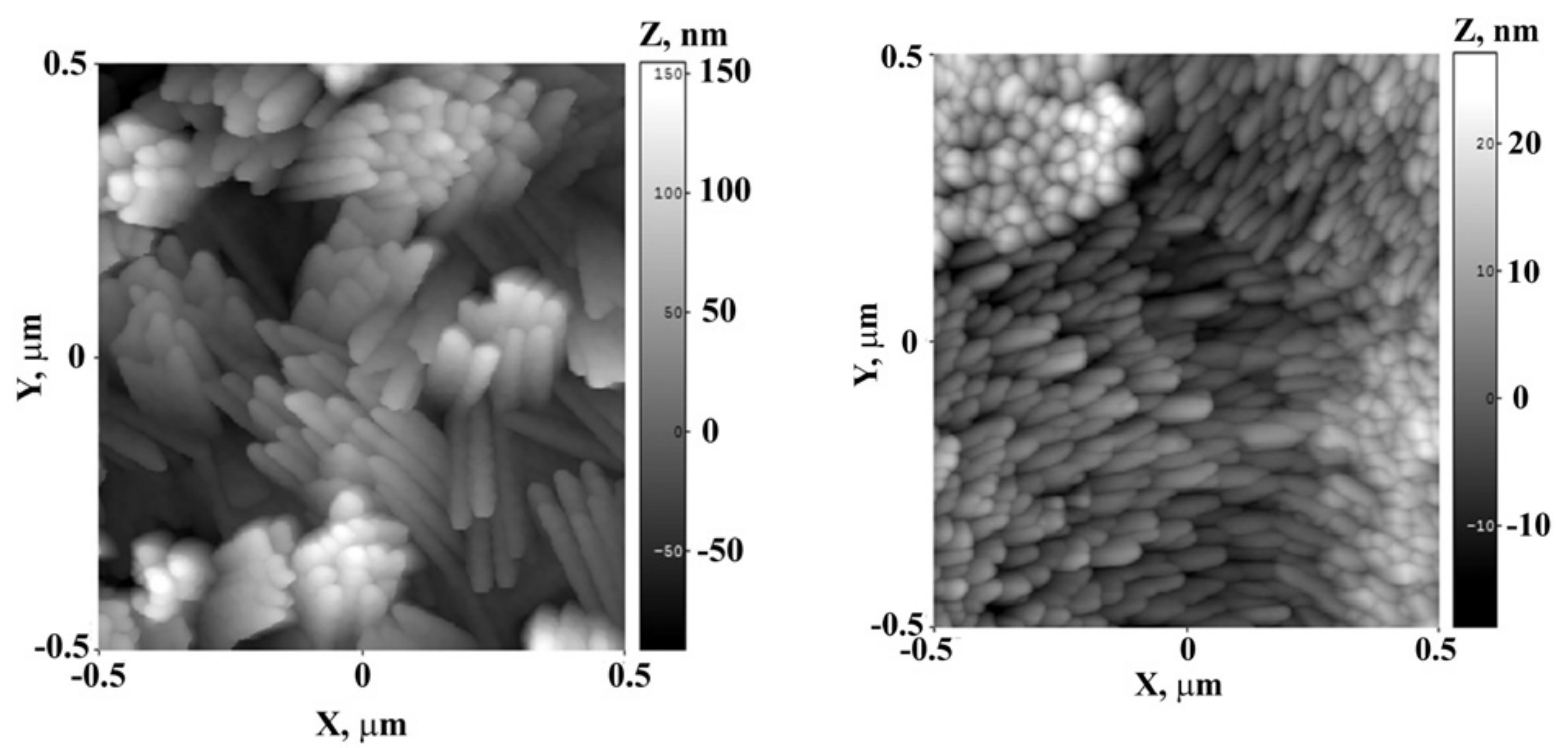

Figure 11. The atomic force microscopy (AFM) images of $150 \mathrm{~nm}$ thick Tellurium nanostructures, deposited at mechanical vibration frequencies of $50 \mathrm{~Hz}$ (at left) and $4 \mathrm{kHz}$ (at right), [12].

As can be seen from the Fig. 12 the acoustic waves applied on the substrate results in more significant morphological changes in the Tellurium nanostructures in comparison with Selenium 
nanostructures. The Fig. 12 (at left) corresponding to the mechanical vibrations frequency of 50 $\mathrm{Hz}$ demonstrates a graded and highly disoriented nanostructure. On the Fig. 12 (at right) corresponding to the mechanical vibrations frequency of $4 \mathrm{kHz}$ is observed ordered columnar nanostructure.
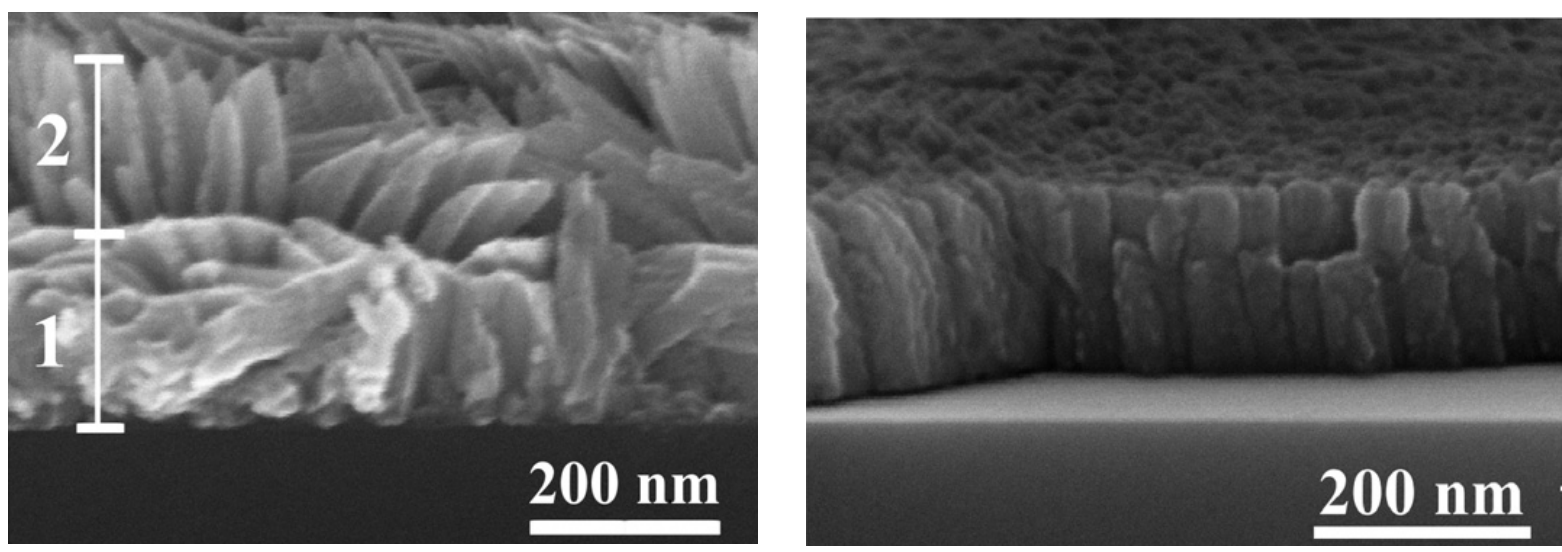

Figure 12. Cross-section scanning electron microscopy (SEM) microphotographs of the Tellurium nanostructures with magnification of $10^{5}$ times. During deposition were applied the following frequencies: $50 \mathrm{~Hz}$, (at left), (1 - dense part; 2 - highly porous part) and 4 kHz, (at right), [12].

\section{Concept of Vibration-Assisted Activation the Pulse-Plasma Deposition Zone}

Two-dimensionally ordered linear-chain carbon nano-matrix also can be considered as both acoustic and electromagnetic sensitive nanostructured metamaterial. The vibration of a sphybridized carbon chains inside the multi-cavity nano-matrix occurs due to the van der Waals interactions between them. Each growed nano-matrix has a unique vibration signature formed by a set of individual CAWs, [15]. As demonstrated by the model experimental systems, vibrational activation is capable of transforming the orientation of nanostructures in the growed nano-matrix.

In the late 18th century, German physicist Chladni demonstrated the organizing power of sound and vibration in a visually striking manner. In the 1950 s the study of wave phenomena was continued by Swiss scientist and anthroposophist Hans Jenny, who named the research field as "Cymatics", ("kyma" is the Greek word for wave), [9]. Under this term, he summarized all phenomena which appear when tone and sound meet the substance. The sound is both a wave and a geometric pattern at the same time. Hans Jenny, too, found that higher frequencies produced more complex shapes. As the frequency rises, the dissolution of one pattern may be 
followed by a short chaotic phase before a new, more intricate, stable structure emerges. If the amplitude is increased, the motions become all the more rapid and turbulent, sometimes producing small eruptions. The shapes, figures and patterns of motion that appeared proved to be primarily a function of frequency, amplitude, and the inherent characteristics of the various materials. An interesting detail in Dr. Jenny's investigations into sound forms in fluids and gases is that if you first produce a disturbance in a fluid, gas or in a flame, then it becomes sensitive to the influence of sound.

The acoustic hologram generated in the nanostructure growth zone through external acoustic activation is capable of controlling the growth process, cluster-assembling and formation of chemical bonds.

In accordance with universal laws of Cymatics and Unified Template (Mereon Matrix) approach, during the vibration-assisted activation of the pulse-plasma deposition region occurs excitation of the self-organized patterns in accordance with 3-dimensional universal template. The Mereon Matrix is a 3 -dimensional template of a dynamic geometric process, $[16,17]$. The structure of material systems is formed on the basis of the universal template, the form of which is defined by presence of vibrations in the system. Connection between shape and vibration determined through the Mereon Matrix.

The unique properties of the nanostructured metamaterials arise not from the properties of the forming initial materials, but from the specific design of their arrangement, geometry and orientation. The geometrical structure of the metamaterials is usually including a repeated pattern at a scale that are smaller than the wavelengths of the phenomena they influence. Nanostructured metamaterials demonstrate unique properties determined by their geometrical structure.

With taking into account the high sensitivity of the structure of a two-dimensionally ordered linear-chain carbon to ultrasonic vibrations [7], we propose the innovative concept, connected with application of the universal Cymatics phenomena and predictive excitation of selforganizing and pattern formation during the vibration-assisted growth of the carbyne-enriched nanostructured metamaterials.

Certain frequencies of acoustic vibrations are capable of forming various geometric shapes. This universal law can be used to program the structure of the grown nano-matrix. Since the carbyne- 
enriched nano-matrix is an acoustically sensitive material, we propose to use acoustic activation of the pulse-plasma zone of nano-matrix growing. At vibrating disturbance onto a pulse-plasma deposition zone will occurs initiation of sensitivity of this medium to the influence of vibrations. In particular, we propose the technology for the patterning and control of the nanostructure growth onto acoustically excited piezoelectric substrates through application of the inverse piezoelectric effect.

Assisting the plasma deposition of the nano-matrixes with acoustic waves leads to patterning phenomena characterized by substantial lateral changes in nanostructure, thickness and properties, [18]. In addition, changes in crystal structure are also induced.

There exist two basic types of the bulk acoustic waves. The first one is the longitudinal wave, in which the oscillations of the particles are only in the direction of the wave propagation. The second one is the shear wave, in which the particle displacements are orthogonal to the wave propagation.

The size and distribution of pattern formation can be controlled through the adjustment of deposition parameters and acoustic wave properties. Changing the acoustic driving frequency can be employed to modify the pattern size.

The piezoelectric elements and layers are also capable of generating electromagnetic radiation, [18]. Acoustic and electromagnetic holograms with specified frequency and spatial characteristics are capable of providing spatial marking of the structure of the grown carbyneenriched nanostructures. The use of combinations of vibrations in different frequency ranges makes it possible to purposefully control the nanostructure of the grown metamaterial.

The new synergistic effect will be provided through the vibration-assisted self-organized wave patterns excitation along with simultaneous manipulating by their properties through the electromagnetic field. Interaction between the inhomogeneous electric field distribution generated on the vibrating layer and the plasma ions will serve as the additional energizing factor controlling the local pattern formation and self-organizing of the nano-structures.

Application of the inverse piezoelectric effect during the ion-assisted carbon pulse-plasma deposition can be provided through the deposited piezoelectric layer, (Fig. 13). The piezoelectric layer converts the released electric energy into mechanical acoustic oscillations that propagating through the substrate and growing nano-matrix. 


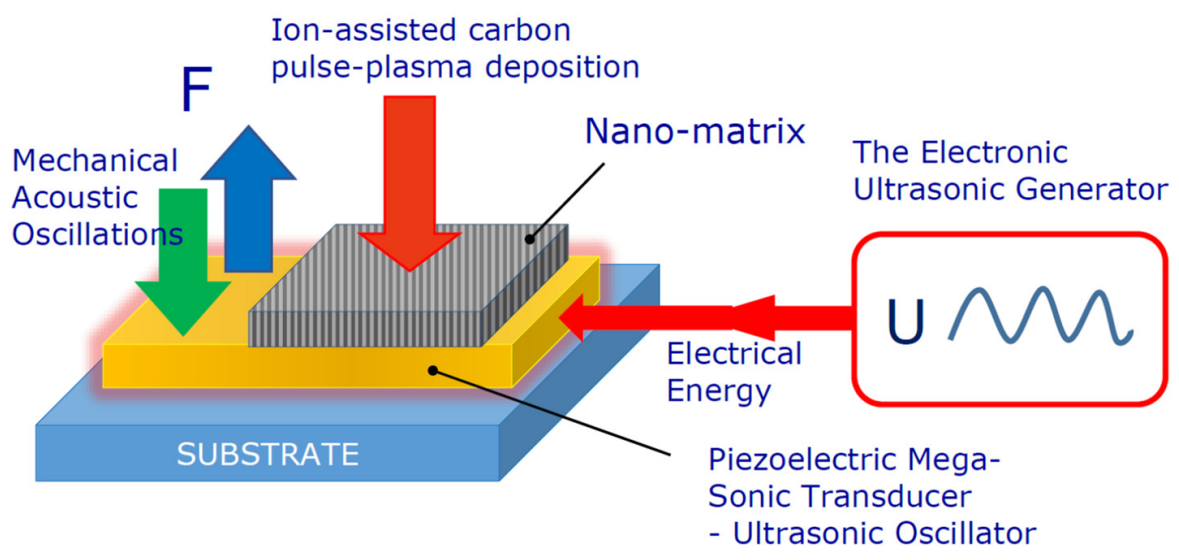

Figure 13. Schematic representation of application of the inverse piezoelectric effect during the ion-assisted carbon pulse-plasma deposition.

Applying an alternating current or radio frequency excitation to electrodes patterned on a piezoelectric material, generates an acoustic wave that propagates in the direction perpendicular to the surface of the deposited nano-structure into the bulk medium (bulk acoustic wave) or along the surface of the deposited nanostructure (surface acoustic wave).

Different acoustic exciting frequencies and waveforms, generated by the piezoelectric layers can be used for programming the required geometric configurations and sizes of the nanostructure of the grown metamaterials. Simultaneous application of the direct and inverse piezoelectric effect opens possibility for interactive ion-assisted pulse-plasma growth the carbon-based nanostructured metamaterials, (Fig. 14).

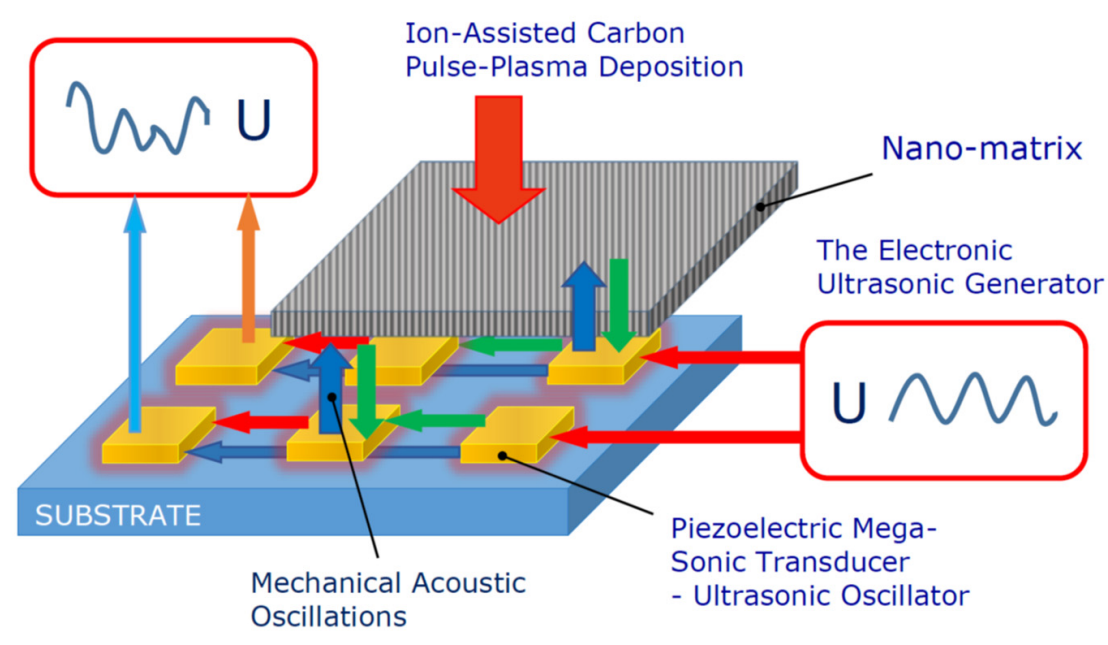

Figure 14. Application of the direct and inverse piezoelectric effect for nano-structural monitoring system during the ion-assisted pulse-plasma deposition. 
Using direct and inverse piezo effect in the nano-structural monitoring system, one piezo transducer is electrically excited to induce vibration which can be detected by a multitude of piezo transducers operated in the sensor mode. By estimating and comparing the excitation signal and the various senor output signals, it is possible to obtain information regarding the current state of the growed nanostructure.

The new fundamental possibilities for predictive programming both by the spatial structure and physics-chemical properties of the functionalized carbyne-enriched nanostructured metamaterials is connected with application of the data-driven carbon nanostructures genome approach. In accordance with the data-driven carbon nanostructures genome approach, for every specific carbon nanostructured metamaterial, there is a specific set of universal relationships like as: "Growth Regimes - Structure - Properties", that allows one to unambiguously predict the main characteristics of the nanostructures under design and the technological regimes required for their predictive growth.

Data-driven genome approach opens new possibilities for programming of the spatially controlled growth, structural self-organizing and cluster-assembling of the two-dimensionally ordered linear chain carbon nano-matrix during the ion-assisted pulse-plasma deposition.

\section{Conclusion}

Two-dimensionally ordered linear chain carbon nano-matrix could serve as an efficient basis for designing and the growth of the new carbyne-enriched nanostructured metamaterials with unique properties. Structural self-organization and pattern formation are the universal and key phenomena observed during growth and cluster-assembling of the carbyne-enriched based nano-matrix at the ion-assisted pulse-plasma deposition. Manipulating by the self-organized patterns excitation we can provide controllable growth and programming of the properties of the functionalized carbyne-enriched nanostructured metamaterials. Fundamental improvement of the ion-assisted pulse-plasma deposition technology for increase stability of the growed carbyne-enriched nano-matrices is connected with application the controllable acoustic activation of the pulse-plasma zone of nano-matrix growing. Acoustic and electromagnetic holograms with specified frequency and spatial characteristics are capable of providing spatial marking of the structure of the grown carbyne-enriched nanostructures. 


\section{Acknowledgments}

The research work is jointly supported and funded by the Scientific and Technological Research Council of Turkey (TÜBITAK) and the Russian Foundation for Basic Research (RFBR) according to the research project № 20-58-46014.

\section{References}

[1]. Heimann, R. B., Evsyukov, S. E., Kavan, L., Carbyne and Carbynoid Structures, Springer Science \& Business Media: Dordrecht, 1999, doil: 10.1007/978-94-011-4742-2

[2]. Hirsch, A., The Era of Carbon Allotropes. Nat. Mater. 2010, 9, 868-871, doi: $10.1038 /$ nmat 2885

[3]. Zhang, K., Zhang, Y., Shi, L., A Review of Linear Carbon Chains, Chinese Chemical Letters, Volume 31, Issue 7, July 2020, Pages 1746-1756, https://doi.org/10.1016/j.cclet.2020.03.019

[4]. Shi, L., Rohringer, P., Suenaga, K. et al. Confined Linear Carbon Chains as a Route to Bulk Carbyne, Nature Mater 15, 634-639, 2016, https://doi.org/10.1038/nmat4617

[5]. Tschannen C.D., et al., Raman Scattering Cross Section of Confined Carbyne, Nano Letters 202020 (9), 6750-6755, DOI: 10.1021/acs.nanolett.0c02632

[6]. Delodovici, F., Carbon sp Chains in Diamond Nanocavities, Phys. Chem. Chem. Phys., 2019, 21, 21814-21823, https://doi.org/10.1039/C9CP03978C

[7]. Flood, P., Babaev, V., Khvostov, V., Novikov, N., Guseva, M., Carbon Material with a Highly Ordered Linear-Chain Structure, In Polyynes Synthesis, Properties, and Applications; Cataldo, F., Ed.; Taylor \& Francis Group: Didcot, UK, 2005, pp. 219-252. https://doi.org/10.1201/9781420027587 
[8]. Arc-Pulse Carbon Plasma Source Technologies, SWISSIMPIANTI Sagl, Switzerland, https://www.swissimpianti.ch/technology

[9]. Jenny, H., Cymatics: A Study of Wave Phenomena \& Vibration, 3rd ed. Macromedia Publishing, New Hampshire, USA, 2001, p. 126.

[10]. Streletskiy, O.A., Zavidovskiy, I.A., Nischak, O.Yu., Dvoryak, S.V., Electrical Conductivity and Structural Properties of a-C:N Films Deposited by lon-Assisted Pulse-Arc Sputtering, Thin Solid Films, Vol. 701, 2020, 137948, https://doi.org/10.1016/j.tsf.2020.137948.

[11]. Streletskiy O.A., Zavidovskiy I.A., Nischak O.Y., Haidarov A.A., Size Control of Silver Nanoclusters During Ion-Assisted Pulse-Plasma Deposition of Carbon-Silver Composite Thin Films, Vacuum, 2020, vol 175, pp. 109286, https://doi.org/10.1016/j.vacuum.2020.109286

[12]. Hristova-Vasileva, T., et al, "Cymatics" of Selenium and Tellurium Films Deposited in Vacuum on Vibrating Substrates, Surface and Coatings Technology, Vol. 307, Part A, 2016, pp. 542-546, https://doi.org/10.1016/j.surfcoat.2016.09.042.

[13] Taillan C., Combe,N., Morillo, J., Nanoscale Self-Organization Using Standing Surface Acoustic Waves, Phys. Rev. Lett. 106 (2011) 076102, doi: 10.1103/PhysRevLett.106.076102

[14] Taillan, C., Combe, N., Morillo, J., Chladni Figures at the Nanoscale, European Physical Journal B: Condensed Matter and Complex Systems, 2015, 88 (12), pp.1-14, doi: 10.1140/epjb/e2015-60695-X

[15]. Ding, D., et. al, The Vibration of a Linear Carbon Chain in Carbon Nanotubes. Materials (Basel, Switzerland), 10(5), 478, 2017, https://doi.org/10.3390/ma10050478

[16]. The Mereon Matrix: Unity, Perspective and Paradox (Elsevier Insights), 2013 1st Ed., ed L Dennis, J B McNair, L H Kauffman p 602. 
[17]. The Mereon Matrix: Everything Connected Through (K)nothing 2018 2nd Ed, ed J B Mcnair, L Dennis, L H Kauffman, World Scientific Publishing Co Pte Ltd p 950.

[18]. García-Valenzuela, A. et al., Patterning and Control of the Nanostructure in Plasma Thin Films with Acoustic Waves: Mechanical vs. Electrical Polarization Effects, Materials Horizons, Mater. Horiz., Vol. 8, № 2, 2021, pp. 515-524, http://dx.doi.org/10.1039/DoMHo1540G 